\title{
IS THE GALACTIC COSMIC-RAY SPECTRUM CONSTANT IN TIME?
}

\author{
David Eichler ${ }^{1}$, Rahul Kumar ${ }^{1}$, AND Martin Pohl ${ }^{2,3}$ \\ ${ }^{1}$ Physics Department, Ben-Gurion University, Be’er-Sheva 84105, Israel; eichler.david@gmail.com, rahuliitk@gmail.com \\ 2 Institut für Physik und Astronomie, Universität Potsdam, D-14476 Potsdam-Golm, Germany; pohlmadq@gmail.com \\ ${ }^{3}$ DESY, D-15738 Zeuthen, Germany \\ Received 2012 December 21; accepted 2013 February 27; published 2013 May 15
}

\begin{abstract}
The hypothesis is considered that the present, local Galactic cosmic-ray spectrum is, due to source intermittency, softer than average over time and over the Galaxy. Measurements of muogenic nuclides underground could provide an independent measurement of the time-averaged spectrum. Source intermittency could also account for the surprising low anisotropy reported by the IceCube Collaboration. Predictions for Galactic emission of ultrahigh-energy (UHE) quanta, such as UHE gamma rays and neutrinos, might be higher or lower than previously estimated.
\end{abstract}

Key word: cosmic rays

Online-only material: color figures

The measured residence spectrum of Galactic cosmic rays $(\mathrm{CRs})$ is $N_{r}(E) d E \propto E^{-2.7} d E$ from $1 \mathrm{GeV}$ to $E_{\mathrm{knee}}=3 \mathrm{PeV}$, and $E^{-3.3}$ from $E_{\text {knee }}$ to $E_{\text {ankle }}=4 \mathrm{EeV}$. This is considerably steeper than the theoretical expectation for the injection spectrum of $N_{i}(E) d E \propto E^{-2.0} d E$ for diffusive shock acceleration by strong shocks. Much of the difference is attributed to the energydependent escape rate $R(E)$ from the Galaxy $R(E) \propto E^{d}$. However, it is questionable whether the entire difference is due to this energy dependence. For one thing, the secondary spectrum is about $N_{s}(E) d E \propto E^{-3.0} d E$ suggesting that $d \sim 0.3$ (Trotta et al. 2011), and, by implication, $N_{i}(E) d E \propto E^{-2.4} d E$. Moreover, if $d$ were much more than 0.3 over many decades of energy, then the anisotropy at the highest energy of Galactic CRs near $1 \mathrm{EeV}$ would be much higher than the reported upper bound of $\sim 10^{-2}$.

There could be many reasons for the injection index to be much steeper than 2.0, but it would in any case be good to know its exact value, as the total energy requirements for Galactic CRs are a sensitive function of it. Moreover, the ultrahighenergy (UHE) emission that could be expected from dense environments that offer a thick target to such CRs is an extremely sensitive function of the injection index. In this paper, we consider the unconventional possibility that the time-averaged residence spectrum is actually flatter than -2.7 by some small amount $\epsilon$. We would then conclude that the injection index is $-(2.4-2 \epsilon)$.

The possibility and consequences of time variability of CRs have received increased attention (Melott \& Thomas 2011; Erlykin \& Wolfendale 2010, and references within) following the suggestions that CR flux correlates with cloud cover (Svensmark et al. 2009) and that very high energy CRs trigger lightning (Gurevich et al. 1992). Erlykin \& Wolfendale have specifically calculated the variation in CR intensity due to discrete, local supernovae at various energies. As far as we know, there is circumstantial evidence that cloud cover, lightning, and CR flux all anti-correlate with solar activity. There is not yet a firm causal link established between CR flux and lightning or cloud cover. The possibility remains that they could all be tied to a fourth correlate; however, it would still be a mystery what this fourth correlate would be. In any case, variations due to terrestrial and heliospheric conditions are separate from variations in the local Galactic flux. This paper is concerned with the latter.
At energies much higher than $1 \mathrm{GeV}, \mathrm{CR}$ production in the Galaxy may be intermittent. Intermittency can lower the anisotropy relative to steady state during the lulls between the intermittent production episodes (Pohl \& Eichler 2011, 2012; Kumar \& Eichler 2012). The recently reported anisotropies and/or upper bounds may be at least partly understandable in these terms. This explanation for the unexpectedly low anisotropy would then predict a slightly steeper present-day spectrum than the time-averaged spectrum.

Abundances of terrestrial cosmogenic nuclides (TCN) can set limits on recent time-averaged variations of the CR flux at Earth, though not on short-term variability. They demonstrate that the CR number flux in the solar system could not have varied on average by more than about $20 \%$ or $30 \%$ of its presently measured value. (Some weak constraint on even short-term $\mathrm{CR}$ variability is placed by the continued existence of life on Earth for hundreds of millions of years. The possibility remains that astrophysically brief fluctuations had interesting terrestrial impact that fell short of total lethality.)

The discreteness of supernovae, assuming they are the main source of CRs, should in any case provide an a priori case for some time variability in the CR flux at Earth. Supernovae are distributed in the plane of the Galaxy, and most local CRs come from sources at a distance commensurate with the halo size, $H$, conventionally taken to be a few kpc. Beyond $H$, CRs preferentially diffuse out of the face of the Galaxy rather than across the disk, and their contribution at large distances, $r$, is exponentially small in $r$ (Pohl \& Eichler 2011).

Fluctuations around the time average should increase as the escape time from the Galaxy decreases. If the escape time decreases with energy, $E$, so then does $N(E)$, and the level of fluctuation increases with $E$. This amounts to fluctuations in the spectral index. Taking for example the rate of supernovae, $P$, the CR source strength, $Q$, and diffusion with diffusion coefficient, $D$, the average CR flux in the Galaxy (of effective radius $R$ ) is

$$
N_{\mathrm{av}}(E) \simeq \frac{Q P \tau_{\mathrm{esc}}}{\pi R^{2} H},
$$

where $\tau_{\text {esc }}=H^{2} / 2 D \simeq 10^{7.5} \mathrm{yr}$ is the escape time. A single source provides CRs that are, let us assume for simplicity, distributed over a sphere of radius $r(t)=\sqrt{D t}$, where $t$ is the time since CR release. For $r(t) \leqslant H$, the density of these 
new CRs therefore is

$$
N_{\mathrm{s}}(E) \simeq \frac{3 Q}{4 \pi r(t)^{3}}=\frac{3 Q}{4 \pi(D t)^{3 / 2}} .
$$

If the supernova is at a distance $r$, one expects a spike in $\mathrm{CR}$ flux of relative amplitude

$$
\eta=\frac{N_{s}}{N_{\mathrm{av}}} \simeq \frac{3 R^{2} H}{4 r^{3} P \tau_{\mathrm{esc}}}
$$

For GeV-band CRs $P \tau_{\text {esc }} \approx 10^{6}$, and therefore we have to be within

$$
r_{c}(\eta) \simeq\left(10^{2} \mathrm{pc}\right) \eta^{-1 / 3}\left(\frac{D(E)}{D(1 \mathrm{GeV})}\right)^{1 / 3} H_{10}^{1 / 3}
$$

of the source to expect a spike of amplitude $\eta$. Note that $r_{c}$ is very weakly dependent on CR energy. The duration of a large fluctuation $(\eta \gtrsim 1) \mathrm{CR}$ event is about

$$
\begin{aligned}
T \simeq & \frac{r_{c}^{2}}{D(E)} \simeq 2 \tau_{\mathrm{esc}} \frac{r_{c}^{2}}{H^{2}} \simeq\left(6 \times 10^{4} \mathrm{yr}\right) \\
& \times\left(\frac{\eta D(E)}{D(1 \mathrm{GeV})}\right)^{-1 / 3} H_{10}^{-4 / 3}
\end{aligned}
$$

The frequency of such CR events is

$$
f \simeq P \frac{r_{c}^{2}}{R^{2}} \simeq 10^{-4} P\left(\frac{D(E) H_{10}}{\eta D(1 \mathrm{GeV})}\right)^{2 / 3}
$$

and the fraction of time that one can expect to experience an event is given by the product of frequency and duration,

$$
f T \simeq 0.06 \eta^{-1}(P \cdot 100 \mathrm{yr})\left(\frac{D(E)}{D(1 \mathrm{GeV})}\right)^{1 / 3} H_{10}^{-2 / 3} .
$$

Thus, significant events with $\eta>1$ are somewhat exceptional, but not unexpected. They are likely to be accompanied by spectral distortions because, at a fixed distance from the source, high-energy CRs arrive earlier than low-energy CRs.

In this paper, we consider the possibility that the average residence index in the Galaxy is $-(2.7-\epsilon), \epsilon \sim 10^{-1}$, that the escape rate exponent $\delta$ is in fact $0.3+\epsilon$, and that the time-averaged $\mathrm{CR}$ injection index is $-(2.4-2 \epsilon)$ rather than -2.4 . This time-averaged spectrum is slightly "propped up" by nearby supernovae, which introduce freshly accelerated populations. That the present-day spectrum is then as steep as $E^{-2.7}$, according to the present hypothesis, due to the paucity of recent, nearby supernovae.

We suggest that the variation of the CR spectral index over time in the $\mathrm{GeV}$ to $\mathrm{TeV}$ range could affect at detectable levels the rate of muogenic nuclei production in deep rock formations that exceed 3 Myr in age. The depth would need to be large enough to be unaffected by possible uncertainties in the erosion rate at the surface. Variations in the CR intensity (due to nearby supernovae, or due to long-term magnetic field change in the Sun or Earth) within the past 3 Myr could possibly be tested by ${ }^{10} \mathrm{Be}$ abundance, to measure the exposure age-and, at the same geographical location, at depths of at least several meters, to isolate the muogenic component. Alternatively, measurements deep enough to give reliable exposure ages, independent of uncertainties in erosion rates, would give an absolute measurement of the past muon flux. The cross section for muogenic ${ }^{53} \mathrm{Mn}$ (half-life 3.7 Myr, exponential decay time 5.3 Myr) from an iron target is $3.8 \mathrm{mb}$ at $E_{\mu}=190 \mathrm{GeV}$. (Heisinger et al. 2002). The production rate per target atom, with a muon flux that is the same as at the sea level, is then $10^{-21} \mathrm{yr}^{-1}$ (Equation (17) of Heisinger et al. 2002). Here we have allowed for the fact that the mean energy at sea level is only $\sim 10 \mathrm{GeV}$, and that the cross section for muogenic ${ }^{53} \mathrm{Mn}$ production varies as $\sim E_{\mu}^{0.86}$. The production flux remains within an order of magnitude of the sea-level value down to depths of $10^{4} \mathrm{~g} \mathrm{~cm}^{-2}$, and analysis of the ${ }^{53} \mathrm{Mn}$ content of subterranean iron ore would give a straightforward measurement of the exponentially weighted average muon flux over the past $5 \mathrm{Myr}$. Comparison with muogenic ${ }^{10} \mathrm{Be}$ (half-life $1.6 \mathrm{Myr}$, production cross section on $\mathrm{O}$ at $190 \mathrm{GeV}$ of $0.094 \mathrm{mb}$ ) could help date the contribution of recent nearby supernovae within the residence time in the disk ( $\sim 3 \mathrm{Myr})$.

Measuring changes in the $\mathrm{CR}$ spectrum that are restricted to energy range $E_{p} \gg 1 \mathrm{TeV}$ would be more difficult, so a time-averaged CR flux that is well above the present only at $E_{p} \geqslant 1 \mathrm{TeV}$ (such as might result from CR injection by ultrarelativistic shocks with Lorentz factors exceeding 100) would be hard to rule out. Muons from primaries at $E_{p} \gg$ $1 \mathrm{TeV}$ dominate the total flux only at depths of $\gtrsim 10^{5} \mathrm{~g} \mathrm{~cm}^{-2}$, where the nuclear muogenesis rate per target atom is only about $10^{-23} \mathrm{yr}^{-1}$. A net collection of $10^{6}{ }^{53} \mathrm{Mn}$ atoms, accumulating over $10^{6.5} \mathrm{yr}$, would thus require a purified sample of $10^{22.5}$ iron nuclei, i.e., several grams.

The dependence of muon secondary flux, and its depth dependence, can be estimated with a simple analytic model: muons at sea level come mainly from primaries at $E \gtrsim 100 \mathrm{GeV}$, which create center-of-mass Lorentz factors that are larger than the ratio of the muon flight time to its rest-frame lifetime. A detailed account of the physics is given in Dorman (2004), and a look-up table for muon production as a function of primary energy is given by Atri \& Melott (2011); for the following discussion, a rough analytic estimate will suffice, and we take the atmosphere to be isothermal and planar: as primaries create the first generation muons at about one interaction depth which we take to be $85 \mathrm{~g} \mathrm{~cm}^{-2}$ in air, or about $1 / 12$ of the total vertical grammage, $1030 \mathrm{~g} \mathrm{~cm}^{-2}$, of the atmosphere, this typically occurs at an altitude of $h_{*} \sim 18 / \cos \theta \mathrm{km}$, where $\theta$ is the angle of incidence of the primary relative to the zenith.

From the muon production altitude $h_{*}$ to altitude $h$, the proper flight time in the muon rest frame is given by

$$
\tau(\theta, h)=\int_{h_{*}}^{h} d \tau=\int_{h_{*}}^{h}-d h^{\prime} / \gamma\left(h^{\prime}, \theta\right) c \cos \theta,
$$

where

$$
\begin{aligned}
\gamma(h, \theta)= & \gamma\left(h_{*}\right)-20 G(h, \theta) / G(0,0)=\gamma\left(h_{*}\right)-20 \\
& \times\left[\int_{h}^{h_{*}} \rho\left(h^{\prime}\right) d h^{\prime} / \cos \theta\right] / G(0,0) .
\end{aligned}
$$

The above expression assumes that muons lose 20 times their rest energy, $1.05 \times 10^{8} \mathrm{eV}$, per vertical atmospheric grammage $G(0,0)$-i.e., about $2 \mathrm{MeV}$ per gram over $1.03 \times 10^{3} \mathrm{~g} \mathrm{~cm}^{-2}$. Assuming a density distribution of $\rho(h)=\rho_{o} \exp \left[-h / h_{s}\right]$, neglecting terms of order $\exp \left[-h_{*} / h_{s}\right]$, and integrating Equation (8) from $h_{*}$ to altitude $h, h \ll h_{*}$, we obtain

$$
\tau(\theta, h)=\frac{h_{s} / c}{\gamma_{*} \cos \theta} \ln \frac{\left[e^{h_{*} / h_{s}} \gamma_{*} \cos \theta-20\right]}{\left[e^{h / h_{s}} \gamma_{*} \cos \theta-20\right]},
$$




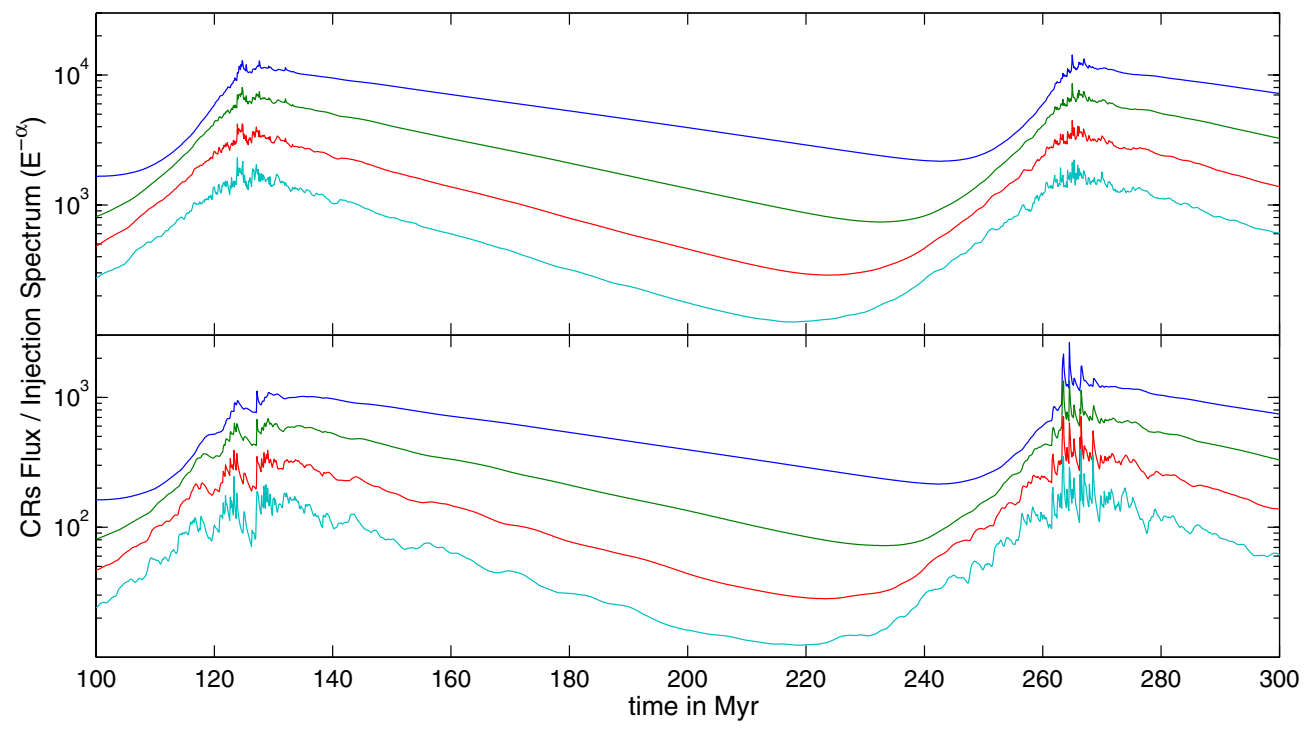

Figure 1. CR flux variation at four different energies for isotropic diffusion in disk geometry, as Sun crosses spiral arms at about $120 \mathrm{Myr}$ (relative time) and $260 \mathrm{Myr}$. Diffusion coefficient for CRs of energy $E$ is taken to be $6 \times 10^{27}(E / 1 \mathrm{GeV})^{1 / 3} \mathrm{~cm}^{2} \mathrm{~s}^{-1}$ and disk boundary is at $1 \mathrm{Kpc}$. Top and bottom panels are for source rates $10^{-2}$ and $10^{-3} \mathrm{yr}^{-1}$, respectively. The blue, green, red, and cyan lines (top to bottom) are for $1 \mathrm{GeV}, 10 \mathrm{GeV}, 100 \mathrm{GeV}$, and $1 \mathrm{TeV}$, respectively, in each panel.

(A color version of this figure is available in the online journal.)

where $\gamma_{*} \equiv \gamma\left(h_{*}\right)$. For $h_{s} \sim 8 \mathrm{~km}$, a reasonable value for Earth's atmosphere over the lowest three scale heights, we can write $h_{s} / c \sim 12 \tau_{\mu}$, where $\tau_{\mu}$ is the muon lifetime. The decay factor is then

$$
e^{-\tau(h, \theta) / \tau_{\mu}}=\left[\frac{\left(\left(e^{h / h_{s}}-20 / \gamma_{*} \cos \theta\right)\right.}{\left(e^{h_{*} / h_{s}}-20 / \gamma_{*} \cos \theta\right)}\right]^{12 / \gamma_{*} \cos \theta} .
$$

The muon flux at sea level, $F_{\mu}(\gamma(0), \theta, 0)$, can be expressed as

$$
\frac{d^{2} F_{\mu}(\gamma, \mu, 0)}{d \gamma d \mu} d \gamma d \mu=\frac{d^{2} F_{\mu}\left(\gamma_{*}, \mu, h_{*}\right)}{d \gamma_{*} d \mu} e^{-\tau(\mu, 0) / \tau_{\mu}} d \gamma_{*} d \mu
$$

where $\mu \equiv \cos \theta$. Integrating over $\mu$ and over $\gamma_{*}$, over the range where $\gamma_{*} \gtrsim 20 / \mu$, and assuming that the muons have a spectrum of $d^{2} F_{\mu}\left(\gamma_{*}, \mu, h_{*}\right) /(d \gamma d \mu) \simeq$ $d^{2} F_{\mu}\left(1, \mu, h_{*}\right) /(d \gamma d \mu) \gamma_{*}^{-2-p} \equiv C \gamma_{*}^{-2-p}$, we obtain for the all-sky muon flux at sea level, $F_{\mu}$,

$$
\begin{aligned}
F_{\mu}(0) \simeq & C \int_{0}^{1} d \cos \theta \int_{20 / \cos \theta}^{\infty} \gamma_{*}^{-2-p} \\
& \times\left[\frac{\left(1-20 / \gamma_{*} \cos \theta\right)}{\left(e^{h / h_{s}}-20 / \gamma_{*} \cos \theta\right)}\right]^{12 / \gamma_{*} \cos \theta} d \gamma_{*},
\end{aligned}
$$

where the lower limit of the integral is determined by the condition that the integrand be positive.

When $\gamma_{*} \gg h_{*} / c \tau_{\mu} \cos \theta \sim 27 / \cos \theta, \tau(h, \theta) / \tau_{\mu} \ll 1$, and the decay factor is about unity. Making the approximation that the decay factor is close to 0 when $\gamma_{*} \leqslant h_{*} / h_{s} \sim 27 / \cos \theta$, and 1 when $\gamma_{*} \geqslant 27 / \cos \theta$, we can estimate the above integral as

$$
\begin{aligned}
F_{\mu}(0) & \simeq C \int_{0}^{1} d \cos \theta \int_{27 / \cos \theta}^{\infty} \gamma^{-2-p} d \gamma_{*} \\
& =\left[1 /(p+1)(p+2)(27)^{p+1}\right] C .
\end{aligned}
$$

Thus, decreasing $p$ from 0.7 to 0.6 with $\left(d F_{\mu}\right) /\left(d \gamma_{*}\right)(1 \mathrm{GeV})$ remaining constant, increases the flux near sea level, $g \equiv$ $G(h, 0) / G(0,0) \sim 1$, by a factor of $(27)^{0.1}(1.7 / 1.6)(2.7 / 2.6) \sim$ 1.53. This estimate is accurate to $10 \%$. The value obtained with the look-up table of Atri \& Melott (2011) gives an increase in the muon flux at sea level of 1.39. The analytic estimate is sufficient for considering the general feasibility of using deep muogenic isotopes to measure the past $\mathrm{CR}$ spectrum.

Deep underground, where $g \gg 27 / 20$, the incident muons need to begin with a minimum $\gamma_{*}$ of $20 \mathrm{~g} / \cos \theta$ in order to survive energy loss, even though their decay is negligible. The flux can then be approximated as

$$
F_{\mu}(g) \simeq C \int_{0}^{1} d \cos \theta \int_{20 g / \cos \theta}^{\infty} \gamma_{*}^{-2-p} d \gamma_{*} .
$$

A change in the spectral index $-2-p$ from -2.7 to -2.6 then leads to a change in the all-sky muon flux of $(20 g)^{0.1}(1.7 / 1.6)(2.7 / 2.6) \sim 1.5 g^{0.1}$.

We now consider how much the observed spectral index is likely to vary with time at Earth. We have calculated an expected intensity versus time plot for randomly placed CR sources in the Galaxy, weighted according to location in proportion to local starlight production. The two figures (Figures 1 and 2) plot the "effective" escape index (EEI)-i.e., the log of the ratio, as a function of energy, of the CR flux to the source spectrum at the same energy - assuming a value for $d$ of $1 / 3$ and assuming that the CR sources are dominated by bright supernovae. This index differential can vary from -0.2 , within the spiral arm, to -0.45 between spiral arms. This is discussed in more detail elsewhere (R. Kumar \& D. Eichler, in preparation). Over timescales of $3 \mathrm{Myr}$, the variation could be of the order of 0.05 , which would produce a ${ }^{53} \mathrm{Mn}$ anomaly of $\gtrsim 10 \%$. This variation would be detectable if the muon flux averaged over the ${ }^{53} \mathrm{Mn}$ lifetime could be measured to $\sim 10 \%$ accuracy at $g \sim 100$.

The solar system now lies between two major spiral arms but inside a minor spiral arm. There are certainly several nearby, recent supernovae, and they and others may have contributed 


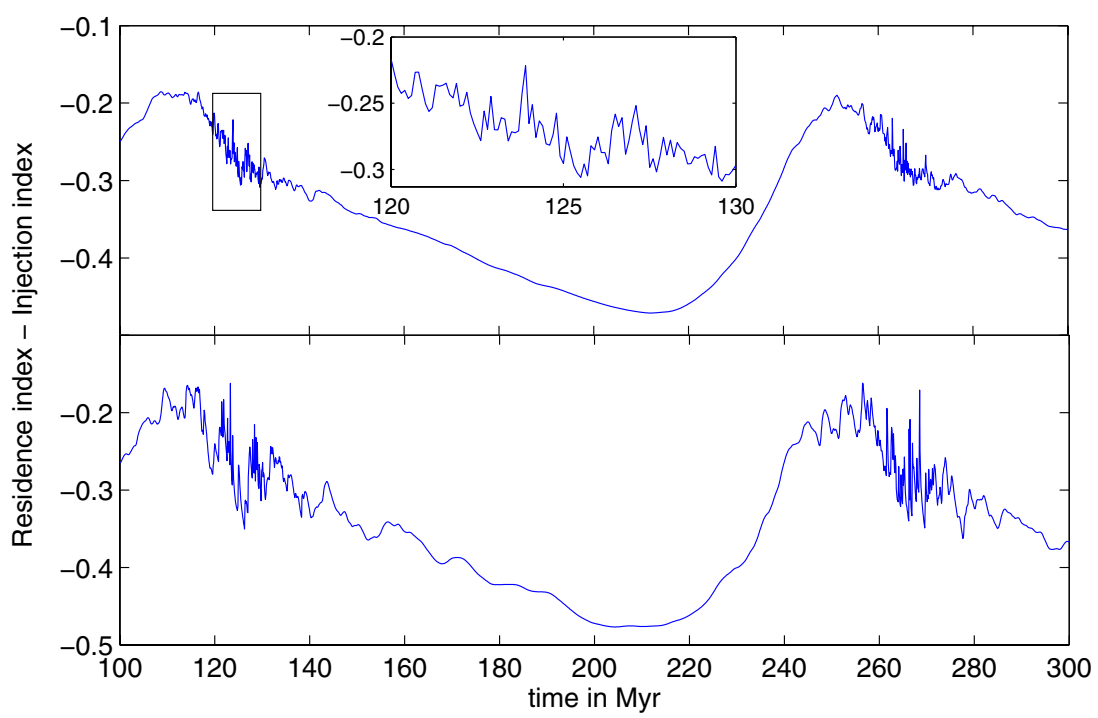

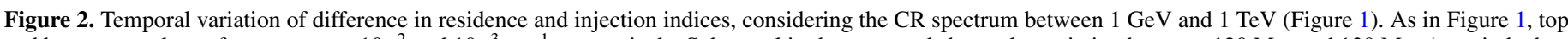

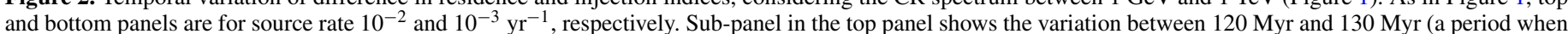
Earth lies just inside a spiral arm) on a smaller timescale (0.1 Myr).

(A color version of this figure is available in the online journal.)

to some fluctuation of the spectral index over the past several million years. In addition, however, the excursion into and out of major spiral arms, as illustrated in the figures, may introduce variations of as much as 0.1 or larger in the effective escape index around its average value. The timescale of this variation is longer than several million years and would not be manifest in ${ }^{53} \mathrm{Mn}$ anomalies, but perhaps would be in longer lived isotopes. If the $\mathrm{CR}$ residence time in the halo is larger than the residence time of the solar system inside a spiral arm, then the timeaveraged value of the EEI is perhaps closer to -0.4 than to -0.3 , and the implied source spectral index is flatter by 0.2 than implied by an EEI of -0.3 . That is, the inferred source spectrum is closer to -2.2 than -2.4 .

The expected emission by the Galaxy of UHE neutrinos, typically generated by primaries in excess of $\sim 10^{14} \mathrm{eV}$, would be raised by about a factor of three for each change of 0.1 in the EEI, and the contrast between the spiral arms and the spaces between them might be enhanced by an even larger factor. According to Figure 1, this factor could be as high as an order of magnitude or more at such high energies. Similar considerations apply to UHE gamma-ray emission from young sources.

The hypothesis that we are living in an unusual era, when the CR flux at high energies is well below its average value in the Galaxy, might be motivated by anthropic considerations: e.g., if a normal CR flux were somehow detrimental to intelligent life. The present level of ionizing radiation from CR flux is less than the component due to terrestrial radon, so it is hard to see how changes of less than a factor of two in the CR flux could have serious astrobiological consequences, though it cannot be ruled out beyond reasonable doubt. At very high energy $\left(\sim 10^{2} \mathrm{PeV}\right)$, intermittency can cause much larger fluctuations in the $\mathrm{CR}$ intensity, and if high-energy airshowers have astrobiological connections (such as lightning, rainfall, and nitrate formation at low altitudes) then it is conceivable that the not unexpected changes in the spectral index of CRs could conceivably affect terrestrial affairs and the development of life and civilization.
Even if very high energy CRs are produced by the same events as lower energy CRs, changes in the spectral index of order 0.1 are not unexpected, and even this modest change could influence the flux at $100 \mathrm{PeV}$ by nearly an order of magnitude.

Neutrino emission from the spiral arms, and UHE gammaray emission from supernovae remnants are sensitive functions of their respective local CR spectral index. For a given gamma-ray flux at $10^{2} \mathrm{MeV}$, they could set useful limits on the spatial variation of the CR spectral index.

We thank Nir Shaviv and Michael Paul for helpful conversations. M.P. acknowledges support by the Helmholtz Alliance for Astroparticle Physics, HAP, funded by the Initiative and Networking Fund of the Helmholtz Association. D.E. and R.K. acknowledge support from the United States-Israel Binational Science Foundation, the Israel Science Foundation, and the Joan and Robert Arnow Chair of Theoretical Astrophysics.

\section{REFERENCES}

Abbasi, R., Abdou, Y., Abu-Zayyad, T., et al. 2010, ApJL, 718, L194

Abbasi, R., Abdou, Y., Abu-Zayyad, T., et al. 2012, ApJ, 746, 33

Atri, D., \& Melott, A. L. 2011, RaPC, 80, 701

Blandford, R. D., \& Eichler, D. E. 1987, PhR, 154, 1

Dorman, L. 2004, Cosmic Rays in the Earth's Atmosphere and Underground (Dordrecht: Kluwer Academic Publishers)

Eichler, D. 1985, ApJ, 294, 40

Ellison, D. C., \& Eichler, D. 1985, PhRL, 55, 273

Erlykin, A. D., \& Wolfendale, A. W. 2010, SGeo, 31, 383

Gurevich, A. V., Milikh, G. M., \& Roussel-Dupre, R. 1992, PhLA, 165,463

Heisinger, B., Lal, D., Jull, A. J. T., et al. 2002, E\&PSL, 200, 345

Kumar, R., \& Eichler, D. 2013, ApJ, submitted

Melott, A., \& Thomas, B. C. 2011, AsBio, 11, 343

Pohl, M., \& Eichler, D. 2011, ApJ, 742, 114

Pohl, M., \& Eichler, D. 2013, ApJ, 766, 4

Svensmark, H., Bondo, T., \& Svensmarks, J. 2009, GeoRL, 36, L15101

Trotta, R., Jóhannesson, G., Moskalenko, I. V., et al. 2011, ApJ, 729, 106 